natur des Zellkerns und der Trophoplastea zusammengestellt.

Die nächsten bejden Abschnitte behandeln den Protoplasten als wässerige Lösung, Emulsion, Suspension, kolloide Lösung, nolekulardisperse Lösung und einfacie Flüssigkeit, und bringen die Auseinandersetzung mit Bütschlis Theorie von der Wabenstruktur des Plasmas.

Den Abschluß der aligemeinen Kapitel bildet eine Eintei ung der mikroskopisch sichtbaren Formelemente der Zelle auf Grundlage ihrer Bedeutung für die Leistung der Zellmaschine und auf Grundlage ibrer Ontogenie:

- I. Der (lebende) Protoplast.

A. Die protoplasmatischen Organe: Zytoplasma, Trophoplasten, Zellkern.

B. Die alioplasmatischen Gebide /durch Umwandling aus protoplasmatischen Organen entstanden).

11. Die ergastischen (toten, vom Protoplasten aufgebuuten) Gebilde.

Den ergatistischen Gebilden, welche als Einschlüsse des Protoplasten auftreten, ist sodann der erste Hauptaischnitt des Bandes gewidmet. Die ergastischen "Ante" - Verfasser bezeichnet damit alle mikrosko pisch kleinen Massenteilchen (von 0,09-100 $\mu$ ) ohne Rïcksicht auf Gestalt, Zusammensetzung und Konsi atenz - können als ergastisch erkannt werden, wenn sie in vorher freien Zellen vollständig neu auftreten oder wenn sie in Reagentien, welche Organsubstanz nicht lüsen, völlig gelöst werden oder wenn sie nur aus chemizchen Substanzen bestehen oder wenn sie kri stallisieren. Vom physiologischen Standpunkte unter scheicel Verfasser Gebrauchsgebilde, Abfallgebilde und Stiitzgribilde, vom topographischen Standpunkte Ein schiuisse und Ausscheidungen. Abfall- und Gebrauchsgebilde künnen unter Uimständen zu ökologischen Zwecken dienen (ökolagische Ante). Es werden so dan der Reibe nach Eiweißante, Kohlehydrate, Fett ante, Abfall- oder Sekretante und Zellsaftante behan delt. Innerbalb der ersten Gruppe sind den Eiweiß krista:len, den Nukleolen und den "Allinanten" be sonders eingenende eigene Lintersuchungen gewidmet. Unter „Aliinanten“ versteht Verfasser „nichtkristallinische, weiche ergratische EiweiBante des Zytoplasmas, welche aus FiwejBkörpern bestimmter mikroskopischer Reaktion, aus Allin, bestehen". Allinante und Cbon driosomen köuucn analoge Gebilde sein; jedenfalls stehen die Eigenschaften der von Benda, Meves, Dues berg zu den Chondriosomen gestellten Gebilde der tierischen Zelle nicht im Widerspruch mit dieser Annabme.

Weitere umfangreiche Eigenuntersuchungen finden sich in iem Kapitel iber Sekretante. An enster Stelle stelit dks in den Chloroplasten auftretende Assimila tionssekret, das früher als Grana oder oltröpfchen beschrieben wurde. Es wird wabrscheinlich als direktes Produkt des Assimilationsprozesses gebildet, und es besteht in der Hauptsache aus dem Hexylenaldehyd $\mathrm{C}_{6} \mathrm{H}_{1 n} \mathrm{O}$. Ein ähnliches Sekret findet sich in dem Zytoplasma der Mesophyllzellen, das Mesophyll- oder Mesekret. Diese Mesekrettropfen werden nach Ansicht des Verfassers wesentlich aus Assimilationssekret be reitet, welches in gelöstem Zustand aus den Chloroplasten in das Zytoplasma aufgenommen und in Tropfenform im Zytoplasma abgelagert wird. Vor komrien und Teaktionen sprechen für diese Annahme. Iu gelistem Zustand kann das Mesekret von Zelle zu
Zelle (bis in das Rindenparenchym der Wurzeln) wandern und wieder abgelagert werden. Biologisch ist es wohl chenso wie das Assimilationssekret als Abiallstoff aufzufassen, jedenfalls nicht als Gebrauchsgebilde.

Das let zte Kapitel, das zweite Hauptkapitel, enthält die Beiprechung des Zytoplasmas. Das Zytoplasma ist nuch. deu Lintersuchungen des Verfassers eine optisch (mikroskopisch und ultramikraskopisch) homogene kolioide L.ösung und auch physiologisch eine homogene Fluissigkeit. In ihm sind die ,ergastischen Organstoffe" gelöst, $d$. h. die Stoffe, weiche in amikroskopischer Verteilung vorbanden sind. Außer den ergastisclitn Stoffen sind aber im Wasser, welches das Dispersionsmittel für alle im Zytoplasma amikroskopiscb gelösten Substanzen bildet, noch „Vitiile" gelüst, weiche den [Tnterschied zwischèn der" homogenen Zytopiasmaflüssigkeit und toten, kolloiden wässerigen Lösungen wesentlich verursachen. Verfasser bommt zu dieser Hypothese auf Grund seiner mikroskopischen Untersuchungen über die ergastische Natur der in der Zelle bekannten chemischen Substanzen. Diese Vitüla sind ungemein kleine, aber trotzdem ungeheuer kompliziert gebaute Gebilde. Xhnlich wie ein Molekül als ein System von in Bewegung begriffenen Elektronen aufgefaBt werden darf, stellt sich Verfasser ein Vitül als ein sehr kompliziertes bewegtes System von kleinsten Realitäten vor, die er als "Mionen" bezeichnet. Die Mionen sind vielleicht die Ursache von Energieformen, welche die Eigenartigkeit der Lebenserscheinungen mit hervorrufen (Nervenenergis, physiologische, Lebens-, psychische Energie älterer Autoren). Mionen können neu entstehen durch Zerlrüimmerung von Atomen, wozu dem Protopiarma Energie. die durch Atmungsprozesse frei wird, zur Verïigung steht. Mionen sind nur in der lebenden Zelle existenzfähig. Ais den Bruchstücken der Vitüle einer absterbenden Zelle bilden sich wiederum ohemische (,vitüiogene") Substanzen. - Die Vitülhypothese unterscheidet sich von a.len bis jetzt aufgestellten Hypothesen, in denen kleine Teilchen zur Erklärung der Lebenserscheinungen benutzt werden, gauz wesentlich dodurch, daß sie eine Forderung der mikroskopischen Morphologie der Zelle ist, daB sie nicht zur Eriklärung einzelner Erscheinungen des Zellenlebeas tienen will und daß sie ganz auf dem Boden des Hypotbesengebäudes der Physik bleibt.

In weiteren Alschnitten des Zytoplasmakapitels werden noch verschiedene Einzelheiten behandelt: Struktur des gelıärteten und gefärbten Zytoplasmas, Fixierung und 1 ärbung der Zelle und der in ihr enthaltenen ergastischen Gebilde, Plasmabrücken. Fritz Jürgen Meyer, Braunschweig.

\section{Physiologische Mitteilungen.}

(Aus den Berichten über die gesamte Physiologie.)

Die Beurteilung der indischen Mond- und Rangoonbohnen. (E. Koch, Zeitschr. f. öffentl. Chemie Jg. 26, S. 16-20, 1920.) Nach Verf. Meinung kann man ein so nährstoffreiches Lebensmittel, wie es die Rangoon. bohne ist, zurzeit nicht ohne weiteres ab'ehnen, wenn der Gebalt an HCN nicht übermäBig boch ist und sich beim Kochen der Speise völlig verflüchtjgt. Quali. tativ hat Verf. auf HCN geprüft, indem er $5 \mathrm{~g}$ zermahlene Bohnen mit $10 \mathrm{ccm}$ Wasser etwa 1/2 Stunde lang in einem Kolben auf $40-50^{\circ}$ erwärmte und dann in die Dämpfe Filtrierpapierstreifen einhing, die nacheinander mit einer 0,2-proz. Guajactinktur und einer 0,1-proz. $\mathrm{CuSO}_{4}$-Lösung getränkt worden waren (Reak- 
tion van Sclbönbein). Die Streifen färbten sich bald grünblau. Ebenso wurden Filtrierpapierstreifen, die mit 1-proz. Pikrinsäurelösung und nach dem Trocknen mit einer 10-proz. $\mathrm{Na}_{2} \mathrm{CO}_{3}$-Lösung getränkt worden waren, in Reagenzrohre eingehängt, die mit Bohnenmehl und Wasser beschickt waren. Nach 6-12 Stunden war eine starke Reaktion auf HCN eingetreten, indem sich die gelben Papierstreifen dunkelrostbraun färbten. Die quantitative Bestimmung des HCN geschah mit $50 \mathrm{~g}$ gemah!enen Bohnen, die im verschlos. senen Kolben 12 Stunden mit $250 \mathrm{ccm}$ Wasser gestanden hatten, indem man nach Zusatz von $10 \mathrm{ccm}$ verdünnter $\mathrm{H}_{2} \mathrm{SO}_{4}$ aus dem Wasserbad mittels Wasserdampf $200 \mathrm{ccm}$ abdestilliert. Das Destillat wurde mlt $\mathrm{NH}_{3}$ stark alkalisch gemacht, mit $10 \mathrm{ccm} 1 / 10 \mathrm{n} \cdot \mathrm{AgNO}_{3}$ versetzt und sofort mit $\mathrm{HNO}_{3}$ angesäuert. Nach Beatimmung des Niedersehlags von $\mathrm{AgCN}$ wurde auf $300 \mathrm{ccm}$ aufgefüllt, abfiltriert und in $150 \mathrm{ccm}$ Filtrat unter Zusatz von $5 \mathrm{ccm}$ konzentrierten gesättigten Eisenammoniumsulfats das übersohüssige Ag mit Rhodan zurücktitriert. Bei 3 Proben solcher Bohnen wurden hiernach $0,0294,0,0294$ und $0,0360 \%$ HCN gefunden. Die letzte Probe wurde einer sorgfältigen küchen. mäßigen Zubereitung durch 3-stündiges Kochen ron $100 \mathrm{~g}$ ganzen Rangoonbohnen mit Wasser unterworfen; in dem abgegossenen Bohnenwasser waren nur Spuren HCN, die unter $0,001 \%$ lagen, nachzuweisen. Die gekochten Bohnen wunden einmal mit Wasser gewaschen, dieses weggegossen, die Bohnen zu Brei verrieben und, wie oben angegeben, mit Wasserdampf destilliert. Es war dabei keine Spur HCN nachzuweisen. Rangoonbohnen mit einem Gehalte an HCN von $0,036 \%$ geben somit bei der Zubereitung zum Genusse die HCN restlos $a b$, so daB eine Gesundheitsschädigung nicht zu befürchten ist. Das Bohnenbrühwasser ist zu verwerfen. Die Zusammensetzung der vom Verf. untersuchten Rangoonbohnen war $(\%)$ : Wasser 11,20 , Fett 1,63, N-Substanz 23,19, Rohfaser 6,45, N-freie Extraktstoffe 54,08, Mineralstoffe 3,45. In Frankreich hat man vorgeschlagen, Rangoonbohnen mit einem Gehalte an HCN von $0,02 \%$ zum Handel zuzulassen.

Riihle.

Körperliche Veränderungen im Gefolge von Gemütsbewegungen. $(F, H$. Kooy, Nederlandsch Maand. schrift voor Geneeskunde J.g. 9, Nr. 1, S. 29-44, 1920) Zur Entscheidung der Fragestellung: „Sind die bei Gemütsbewegungen gefundenen Funktionsstörungen psychisch oder körperlich bedingt?" untersuchte Verf. 1. Blutzucker; Resultate mit der Mikromethode von Bang; vor und $3 / 4,11 / 2,21 / 4$ und oft 3 Stunden nach dem Friihstiuck, bestelhend aus $100 \mathrm{~g}$ Brot und $200 \mathrm{ccm}$ Mileh. 20 ruhige Gesunde zeigten die Mittelwerte 0,98; 1,$14 ; 1,16 ; 1,04 ; 1,02 \%$. Ruhige Hebephrene wiesen niedrige Werte auf; eine unruhige, ängstliche Dementia-praecox-Kranke zeigte 4 Stunden nach einem kargen Frühstïck 1,59\%; nach eingetretener Beruhigung 1,33-1,20-1,20-1,07-1,01\% und noch später $0,89 \%$ Ruhige Epileptiker ergaben: 0,9-1,161,04-0,98-0,93\%; eine wütende Epileptica 1,011,58-1,31-1,09-1,13\%. Ein Paralytiker im Zorn: $0,87-1,43-1,22 \%$, an einem ruhigen Tag $0,83-$ 1,16-1,05\%. Ein Psychastheniker in Ruhe: 1,09$1,08-1,09-0,95-1,02 \%$; in Aufregung: $1,02-$ $1,50-1,13-0,92-0,87 \%$ Mittelwerte bei echter, primärer Melancholie (19 Fäl'e): 1,12-1,55-1,39$1,21 \%$; die nicht üngstlichen unter diesen gaben: 1,03-1,39-1,22-1,03\%; die ängstlichen unter ihnen hatten: $1,13-1,63-1,45-1,19 \%$ - 2. Blutdruck: Die bekannten Blutdruckstörungen ibei psychischer Er- regıng jeder Art werden bestätigt. - 3. Temperatur: Erhöhung wird gefunden bei Amentia, akuter Verwirrtheit nach psychischem Trauma unter AusschluB körperlicher Ursachen. - 4. Intestinale Störungen: Verf. nimmt als Beispiel Obstipation bei Melancholischen, bei denen in den angstlichen Perioden die $\mathrm{Ob}$ stipation zunahm, unberinflu日t von Opium und Laxanzien. - Diese körperlichen Erscheinungen, besonders in den Zuständen von Zorn und Angst, werden aufgefaBt als Reaktion des sympathischen Nervensystems, d. h. des thoracico-lumbalen Teils des unwillkürlichen Nervensystems; in der Beweisführung für diese Annahme stützt sich Verf. weitgehend auf Untersuchungen von Gaskell und Cannon, die nicht gesondert referiert werden können. W. Weiland, Hamburg.

Uber Eigenblutinfusion. (A, Döderlein, Dtsch. med. Wochenschr. Jg. 46, Nr. 17, S. 449-451, 1920.) Dödcrlein befürwortet aufs wïrmste die zuerst von Thies empfohlene Eigenblutinfusion zur Bekümpfung des Verblutungstodes bei Blutungen in die Körperhöhlen, wie sie u. a. bei tubargraviden Frauen mit Fruchtkapselaufbruch, bei Milz- oder Leberrupturen u. dgl, in die Bauchböhle vorkommen. Er führt die Reinfusion folgendermaBen aus: Das Blut wird bei Beckentieflagerung aus der Bauchhöhle über eine in den Bauchwand'halter eingefügte Rinne direkt in einen mit Gaze ausgekleideten Trichter aufgefangen, durch den es in einem Erlenmeyerkolben filtriert wird. Dann wird es im Verhältnis von $3: 1$ mit einer 1-proz. Lösung von Natrium citricum vermischt und körperwarm in eine Viene infundiert. Der Ėrolg ist ein verblüffender - ,die totenbleiche Patientin wird in der Tat sichtlich wiederbelebt, die wachsbleichen Lippen färben sich im Augenblick, die Wangen röten sich, der Puls kehrt wieder", und am folmenden und den nitchsten Tagen sind die Kranken, die dem Tode geweiht waren, wieder ganz frisch und munter. Die unliebsamen Nebenerscheinungen, wie Somnolenz, Ikterus, Schüttelfrost und ähnliches, die von einigen Seiten (Opitz, Arnim) beobachtet worden sind, hält $D$. für beleutungslos gegenüber dem lebennettenden Eriolg der Eigenblutinfusion, die er als ein ungefährliches Verfahren bezeichnet. F. v. Krüger, Rostock.

Die Wirkungen des Schilddrissenhormons. (Leon Asher, Therap. Halbmonatsh. Jg. 34, H. 8, S. 222-224, 1920.) Gedrïngte Zusammenfassung unserer derzeitigen Kenntnisse über die Wirkungen des Schilddrüsenhormons. Seine chemische Zusammensetzung ist noch unbekannt; die Untersuchungen Kendalls, der aus der Schilddrüse eine reine Substanz, ein Trijodindolderivat, „Thyrotoxin", mit der vollen Wirksamkeit der Schilddrüse hergesteilt hat, sind noch zu bsstätigen. Schilddrüsengewebe oder Auszüge aus gesunden Schilddrüsen haben scharf umschriebene, spezifische Wirkungen. Im Vordergrund steht eine Steigerung des Stoffiwechsels, die mit Sicherbeit allerdings nur an schilddrüsenlosen Tieren oder an. Menschen erzeugt werden kann, bei denen die Drüse degeneriert ist, also in den Fällen, in denen der Stoffwechsel herabgesetzt ist. Manche anscheinend norma'e Menschen sind gegen das Schilddrüsengift besonders empfindlich; auch bei unversehrten Ratten lassen sich durch mehrtägige Verfütterung von Schilddrüsenpräparaten Erregungssymptome hervorrufen, die in vieler Beziehung an die Baselowsche Krankheit gemahnen. Die Tiere werden sehr lebhaft; die erhöhte Beweglichkeit ist aber nicht die Ursache der Stoffwechselsteigerung. Gegen reinen Sauerstoff- 